\title{
Role of frequency and mechanical index in ultrasonic-enhanced chemotherapy in rats
}

Bryant J. Staples Æ Beverly L. Roeder Æ Ghaleb A. Husseini Æ Odgerel Badamjav Æ G. Bruce Schaalje Æ William G. Pitt

Received: 25 August 2008/Accepted: 15 December 2008/Published online: 7 January 2009

Springer-Verlag 2009

\begin{abstract}
Purpose The therapeutic effect of ultrasound and micellar-encapsulated doxorubicin was studied in vivo using a tumorbearing rat model with emphasis on how tumor growth rate is affected by ultrasonic parameters such as frequency and intensity.

Methods This study employed ultrasound of two different frequencies $(20,476 \mathrm{kHz})$ and two pulse intensities, but identical mechanical indices and temporal average intensities. Ultrasound was applied weekly for 15 min to one of two bilateral leg tumors (DHD/K12/TRb colorectal epithelial cell line) in the rat model immediately after intravenous injection of micelle-encapsulated doxorubicin. This therapy was applied weekly for 6 weeks.

Results Results showed that tumors treated with drug and ultrasound displayed, on average, slower growth rates than noninsonated tumors $(\mathrm{P}=0.0047)$. However, comparison between tumors that received 20 or $476-\mathrm{kHz}$ ultrasound treatments showed no statistical difference $(\mathrm{P}=0.9275)$ in tumor growth rate.
\end{abstract}

Conclusion Application of ultrasound in combination with drug therapy was effective in reducing tumor growth rate, irrespective of which frequency was employed.

Introduction

The use of chemotherapeutic drugs is a common treatment for solid cancerous tumors. Unfortunately, these drugs are often not selective between healthy and cancerous cells. Therefore, it is difficult to optimize the balance between administering enough drug to destroy the cancer and giving so much drug that it causes patient morbidity and mortality. These drawbacks to conventional chemotherapy lead many investigations to seek novel ways to locally deliver the drugs selectively to the tumor, thus eliminating damaging exposure to healthy tissues.

There are several novel nano-sized carriers that have been used for ultrasonic-enhanced drug delivery [1]. These include micelles, liposomes and polymeric nanoparticles.

Micelles are usually used to deliver hydrophobic drugs because they can be easily sequestered in the hydrophobic interior of the micelle [2]. They are usually polymeric in nature, and have been combined with ultrasound (US) to treat solid tumors with anthracycline drugs [3-7]. Liposomes differ from micelles in that their drug payload is usually sequestered in their aqueous interior [8]. Liposomes containing doxorubicin have been combined with ultrasound to treat solid tumors [9-11]. Nanoparticles containing (or consisting of) drugs are usually designed to be small enough to extravasate beyond the endothelial boundary of capillaries [12, 13]. Ultrasound is used to enhance the extravasation process.

Ultrasound has also been used for gene delivery, usually in conjunction with liposomes and polymeric nanoparticles [14-16]. For example, the group of Hosseinkhani has shown effective ultrasonic-enhanced gene delivery using polyplexes of DNA and cationic-derivatized natural polymers, such as cationized gelatin [17-20] and dextran [21]. The authors speculated that cavitation-induced cell membrane damage and permeation were responsible for the enhanced genetic expression.

This paper presents our research in which chemotherapeutic drugs are loaded inside stable micelle carriers and injected into the circulatory system of a rat. Ultrasound is then used to locally deliver the toxic drug by permeabilizing the cell membranes of cancerous tissue while simultaneously disrupting the micellar structure to release the drug [1]. This treatment ideally has the potential both to preclude toxicity away from the target tumor and to locally increase the drug concentration in the cancerous tissue, thus increasing the drug's efficacy. However, specific challenges remain, and the delivery still needs to be 
optimized. Important issues to address include whether different frequencies and intensities of ultrasound affect the delivery of the drug and whether this delivery system is effective in treating cancer [22]. The purpose of this article is to address some of these issues and provide a greater understanding of ultrasonic drug delivery from micelles.

Our research uses stabilized polymeric micelles to carry doxorubicin (Dox) through the blood system of a rat, whose hind legs host colorectal epithelial solid tumors. Ultrasound is then applied to only one of the tumors. Ultrasound consists of pressure waves with frequencies greater than 20 $\mathrm{kHz}$, generated by transducers that change a voltage waveform into mechanical movement of the transducer face. Like optical and audio waves, ultrasonic waves can be focused, reflected, refracted, and propagated through a medium [23]. Therefore, ultrasonic waves can be directed to and/or focused on a specific tissue area-a useful property that makes ultrasonic therapy minimally invasive (no surgery required).

Ultrasound intensity is the amount of power delivered by the pressure wave per unit area, commonly measured in watts per square centimeter $\left(\mathrm{W} / \mathrm{cm}^{2}\right)$. As ultrasonic intensity increases, gas bubbles form and oscillate in the ultrasonic pressure field, a phenomenon called cavitation. At sufficiently high intensities, the bubble oscillations become unstable, and the inertia of the fluid can cause the gas bubble to collapse into a small volume of highly compressed and heated gas [24]. This type of cavitation, called inertial or collapse cavitation, can be stressful to cells because of the very high shear stresses in the region of the collapse, the concentrated energy of the shock wave produced by the bubble collapse, and the free radicals produced by the high temperatures. Furthermore, if the collapse is near a solid surface or even the semi-solid surface of a cell, an asymmetrical collapse occurs, which ejects a jet of liquid at sufficient speed to pierce nearby cells $[25,26]$.

In general, the likelihood and intensity of collapse cavitation increases at higher intensities and lower frequencies [25] and is indicated by the "mechanical index" (MI), the ratio of peak negative pressure, $\mathrm{P}^{-}$, (in $\mathrm{MPa}$ ) to the square root of frequency, $\mathrm{f},($ in $\mathrm{MHz}$ ): [27]

$$
\mathrm{MI}=\frac{P^{-} / \mathrm{MPa}}{\sqrt{f / \mathrm{MHz}}}
$$

The threshold for collapse cavitation occurs at about MI $=0.3$, biological effects are observed at MI $[0.6$, and tissue damage is often observed when MI $>1$ [27, 28].

In the search for better cancer treatment methods, new innovations in micellar drug delivery have emerged with successful preliminary results. A micellar aggregate of polymeric surfactant creates a hydrophobic core and a hydrophilic corona. Micelles carry hydrophobic chemotherapeutic drugs within their cores and can deliver these drugs directly to target areas. In vitro release from our micellar carriers is induced by ultrasound when the MI C $0.38[4,29]$.

Ultrasound has been shown to trigger the release of Dox from Pluronic micelles at low frequencies [5, 7, 30-33]. Munshi et al. [34] were the first to report that ultrasound enhanced the uptake of Dox from micelles to a human leukemia cell line. In vitro studies by Husseini et al. [30] showed that $70 \mathrm{kHz}$ ultrasound released about $10 \%$ of the drug from the micelles, and that after cessation of insonation, the drug was quickly re-sequestered in the micelle. Marin et al. studied the uptake and distribution of doxorubicin released from Pluronic micelles. They concluded that there are two different mechanisms involved. First, ultrasound releases the drug from the micelles, causing a higher local concentration than observed without ultrasound. Second, ultrasound perturbed the cell membranes, which resulted in more accumulation of drug inside the cells [33].

Nelson et al. [35] employed an in vivo rat model to investigate the effects of ultrasonically controlled release of micelle-encapsulated doxorubicin. The particular micellar carrier used in that work consisted of polyether triblock surfactants stabilized by an interpenetrating network of a thermally sensitive acrylamide [36]. In that study, Dox in the carrier and ultrasound were applied weekly for 4 weeks. The tumors were exposed to 20 or $70-\mathrm{kHz}$ ultrasound for $1 \mathrm{~h}$ following each weekly dose. Results showed that application of low-frequency ultrasound and encapsulated Dox at concentrations of $2.67 \mathrm{mg} / \mathrm{kg}$ resulted in a significant decrease in tumor size compared to controls [35].

The present study goes beyond Nelson's experiments to address some significant unresolved issues and to identify possible mechanisms. Specifically, our study held the mechanical index and time-averaged power intensity constant in order to solely investigate the effect of frequency on the therapeutic effect of Dox from stabilized micelles.

\section{Materials and methods}

In this experiment, 24 rats were treated for six consecutive weeks. Once a week, each rat received a systemic injection of micellar-encapsulated Dox followed by ultrasound treatment. All procedures involving rats followed NIH 
guidelines for humane animal use and care, and were approved by IACUC of Brigham Young University.

Tumor model

The BDIX rat readily grows the $\mathrm{DHD} / \mathrm{K} 12 / \mathrm{TRb}$ colorectal epithelial cancer cell line, which is susceptible to Dox [37]. This cell line can be injected nearly anywhere in the rat to successfully produce tumors [38]. Tumors were grown by intradermal injection of 2511 of cells $\left(2910^{6} \mathrm{cell} / \mathrm{ml}\right)$ in both rear legs of 24 female BDIX/CRCrl rats (Charles River Laboratories, Wilmington, MA) as described in Nelson et al. [35]. Tumors were apparent at the inoculation site within 2-3 weeks.

\section{Drug/micelle preparation}

The drug carrying micelles used herein were stabilized Pluronic P105 micelles that were synthesized as described previously and stored at -20C until use [36]. These micelles, called NanoDeliv ${ }^{\mathrm{TM}}$, consist of a hydrophobic core of polypropylene oxide and an outer corona of polyethylene oxide, stabilized using an interpenetrating network of thermally responsive acrylamide. At room temperature, the average diameter is about $125 \mathrm{~nm}$. Doxorubicin was loaded into these micelles by introducing

$3.75 \mathrm{ml}$ of stabilized micelle suspension into a $10-\mathrm{mg}$ vial of Dox via a $0.22-1 m$ membrane filter (for sterilization). The Dox/micelle suspension was stored at $-20 \mathrm{C}$ and only thawed for short periods at the time of injection.

Encapsulated Dox injection

Prior to Dox injection, rats were tranquilized initially with ketamine $(55 \mathrm{mg} / \mathrm{kg}$, IP) and then pretreated with dexamethasone $(4.0 \mathrm{mg} / \mathrm{kg}, \mathrm{SC})$ and diphenhydramine $(5.0$ $\mathrm{mg} / \mathrm{kg}, \mathrm{SC}$ ) injections to reduce the risk of anaphylactic shock. The hind legs and tail were shaved, and the hair at the tumor site receiving ultrasound was completely removed through application of depilatory cream (Nair) for $60 \mathrm{~s}$. Lubricating ophthalmic ointment was administered to prevent the rats' eyes from drying during the anesthetic period.

Administration of the encapsulated Dox at $2.67 \mathrm{mg} / \mathrm{kg}$ (rat body mass) was given via a $25 \mathrm{~g}$ needle winged infusion set (Terumo, Somerset, NJ) in the lateral tail vein, followed by $3 \mathrm{ml}$ of physiologic saline to completely flush the drug from the catheter. Rats were immediately placed in ventral recumbency and observed for adequacy of breathing.

Ultrasound application
Following the injection of Dox, the rats were sedated with the alpha- 2 agonist medetomidine $(0.3 \mathrm{mg} / \mathrm{kg}$, IP), which when combined with the previously administered ketamine, produced adequate sedation and muscle relaxation for the procedure. Medetomidine administration was necessary to prevent the rats from moving their legs while ultrasound was applied to the tumor.

Approximately 5 min after infusion, ultrasound treatment was applied for $15 \mathrm{~min}$ to one tumor using either a $20-\mathrm{kHz}$ probe (Vibra-Cell; Sonics \& Materials, Inc., Newtown, CT) or a $476-\mathrm{kHz}$ transducer (Sonic Concepts, Woodinville, WA). The second tumor (on the contralateral leg) was not exposed to ultrasound and thus served as the internal control. Half of the rats were insonated using the $20-\mathrm{kHz}$ probe (continuous wave, intensity of $1.0 \mathrm{~W} / \mathrm{cm}^{2}$, pressure amplitude of $0.173 \mathrm{MPa}$ ). The mechanical index at the tip of the $20-\mathrm{kHz}$ transducer operating at $1.0 \mathrm{~W} / \mathrm{cm}^{2}$ was 1.22 . The other 12 rats were treated using the $476-\mathrm{kHz}$ transducer. In order to match the mechanical index (1.22) while using 476$\mathrm{kHz}$ US, an intensity of $23.61 \mathrm{~W} / \mathrm{cm}^{2}$ (pressure amplitude of $0.842 \mathrm{MPa}$ ) was required. However, transmitting this amount of energy would most likely cause thermal damage to the rat tissue. Therefore, the $476-\mathrm{kHz}$ transducer was pulsed $(1,000$ cycle) at a pulse repetition frequency of $20.161 \mathrm{~Hz}$ (duty cycle of 0.0424 ) to create a temporal average intensity of $1.0 \mathrm{~W} / \mathrm{cm}^{2}$. Thus both ultrasonic applications had the same mechanical index of 1.22 and the same temporal average power density of $1.0 \mathrm{~W} / \mathrm{cm}^{2}$.

For insonation at $20 \mathrm{kHz}$, ultrasound-conducting gel (Aquasonic 100, Parker Laboratories, Fairfield, NJ) was applied on the skin of the leg above the tumor, and the $20 \mathrm{kHz}$ probe was placed in this gel but not directly touching the skin. For insonation at $476 \mathrm{kHz}$, ultrasound-conducting gel was applied on an acoustically transparent window of polypropylene film (38 lm thick, Exxon Chemical, MarLin, PA) at the focal point of the $476-\mathrm{kHz}$ transducer. The targeted tumor was placed in the gel at the focal point, and the leg was immobilized with tape applied at the tarsus below the tumor site.

Tumor growth measurement

After 15 min of insonation, the tumor sizes were measured, and the rats were injected subcutaneously with atipamizole $(84.0 \mathrm{ll} / \mathrm{kg})$, to reverse the effect of medetomidine. Each tumor was measured by making two perpendicular measurements ( $\mathrm{a}$ and $\mathrm{b}$, with $\mathrm{a} \mathrm{C}$ b) using calipers. Tumor volume (TV) was then determined using the formula [38]: a $b^{2}$

TV $1 / 4$ 
2

In some cases, it became difficult to accurately measure the size of the tumor. Skin covering the area of interest made it difficult to determine the exact edges of the solid mass. Therefore, each measurement was taken three times to help decrease this "noise" and increase the reliability of the statistical analysis.

\section{Statistical procedure}

The tumor volume data (from insonated and control tumors) were analyzed using an exponential growth model:

$\operatorname{TV} 1 / 4 \mathrm{~A}_{0} \mathrm{e}^{\mathrm{kt}}$

where $t$ is the time after the first drug injection, TV is the tumor volume at time $\mathrm{t}, \mathrm{A}_{0}$ is the tumor volume immediately following the first treatment $(\mathrm{t}=0)$, and $\mathrm{k}$ is the tumor growth rate constant.

Tumor volumes were transformed to the natural logarithmic scale:

$\ln$ TVp $1 / 4 \ln ð \mathrm{~A}_{0} \mathrm{p} \mathrm{p} \mathrm{kt}$

Using SAS software (SAS Institute Inc., Cary, NC), logtransformed volumes were then analyzed using a linear mixed model with ultrasound treatment, frequency, days after initiation of treatment, and all possible interaction of these factors as fixed effects. Initial tumor volumes, ratspecific tumor growth rates, and repeated volume determinations were considered to be random effects. The model was fitted using residual maximum likelihood for the variance components and estimated generalized least squares for the fixed effects. Residuals were computed and plotted versus predicted values to assess goodness-offit of the model. Main effects and interactions of the fixed effects were tested using approximate $F$ tests based on the Kenward-Roger adjustment for small-sample inferences.

Results and discussion

Our in vivo rat model was employed to investigate a novel tumor treatment involving the localized delivery of a chemotherapeutic drug (Dox) using stabilized Pluronic micelles as the drug carrier and low-frequency ultrasound as an external triggering mechanism to release the drug directly at the treatment site. As part of this research, the effects upon tumor growth were successfully studied using different ultrasonic frequencies but identical values for intensity and MI.
Of the 24 rats initially used in the experiment, 23 survived all 6 weeks of the study. One rat from the $476-\mathrm{kHz}$ group died during the first day of treatment. Autopsy results for the rat that died implied hypothermia to be the cause of death, not the treatment or the cancer. The hypothermia was thought to have occurred because the rat was not completely dried after being washed to remove the depilatory lotion. As the rat was under anesthesia, its ability to control body temperature was compromised. There were no other complications during the experiment. Most of the rats, however, became lethargic and started to lose substantial weight (greater than $10 \%$ over a week) after the fifth week of treatment. It is suspected that this was due to the combination of the chemotherapy and the growing tumors, some of which had metastasized to other locations. Necropsy on a subset of the rats showed that all animals sampled had metastatic lesions in the lungs and abdominal lymphatic system ( 9 of 9), irrespective of whether they received 20 or $476-\mathrm{kHz}$ US.

The tumor growth (or recession) rate was adequately modeled by an exponential growth rate, for both control and insonated tumors at both frequencies. Comparison between tumors that received $20-\mathrm{kHz}$ US and those that received $476-\mathrm{kHz}$ ultrasound showed no statistical difference ( $\mathrm{P}=$ 0.9275 ) in tumor growth rate. We could not reject the null hypothesis that the tumors grew at the same rate with 20 and 476-kHz insonation.

Because there was no difference attributed to the parameter of frequency, the data from both frequencies were combined to examine the differences in growth rate for the insonated tumors versus control tumors. The exponential growth rate constant for the non-insonated tumors $\left(\mathrm{k}_{\mathrm{control}}\right)$ was $0.0465 /$ day (standard error $=0.0066$ ), while that for both frequencies of insonated tumors

( $\mathrm{k}_{\text {ultrasound }}$ ) was $0.0402 /$ day (standard error $=0.0066$ ). The difference was $0.0063 /$ day (standard error $=0.0022$ ). Comparison between the growth rate constants of the insonated tumors and non-insonated tumors showed that the insonated tumors displayed significantly slower growth rate $(\mathrm{P}=0.0047)$. The percent growth inhibition, defined as

$\left\{1-\mathrm{k}_{\text {insonated }} / \mathrm{k}_{\text {control }}\right\} 9100$, was $13.5 \%$. Figure 1 shows an example of tumor growth data collected from one of the rats in the study (from the $476-\mathrm{kHz}$ group). These particular rat data were chosen because they are representative of the statistical average results of the study. Within the group that received $20-\mathrm{kHz}$ US, the treated growth rate constant was $0.048 \pm 0.007 /$ day for treated tumors and

$0.053 \pm 0.005 /$ day for untreated tumors. Within the group that received $476-\mathrm{kHz}$ US, the treated growth rate constant 
was $0.032 \pm 0.013$ /day for treated tumors and $0.038 \pm$ $0.007 /$ day for untreated tumors.

In addition to the overall group comparisons described above, a paired comparison statistical analysis was done in which the growth rate of the treated tumor was compared directly to the growth rate of the contralateral untreated tumor in the same rat. This analysis showed that for rats in the $20-\mathrm{kHz}$ group, the hypothesis that the insonated tumors grew at the same rate was very close to the boundary of being rejected $(\mathrm{P}=0.057)$. However, in the $476-\mathrm{kHz}$ group, we could reject the null hypothesis and state that the treated tumor grew slower than the contralateral noninsonated control in the same rat $(\mathrm{P}=0.028)$. When combining both groups, the treated tumor definitely grew slower than the contralateral control in the same rat $(\mathrm{P}=0.006)$. This is consistent with other similar studies in rats and mice [3, 35, 39].

For example, in a similar study Nelson et al. [35] used the same in vivo rat model to investigate the effects of ultrasonically controlled release of micelle-encapsulated Dox. During the course of their 4-week treatment, the volumes were measured for tumors exposed to 20 or $70-\mathrm{kHz}$

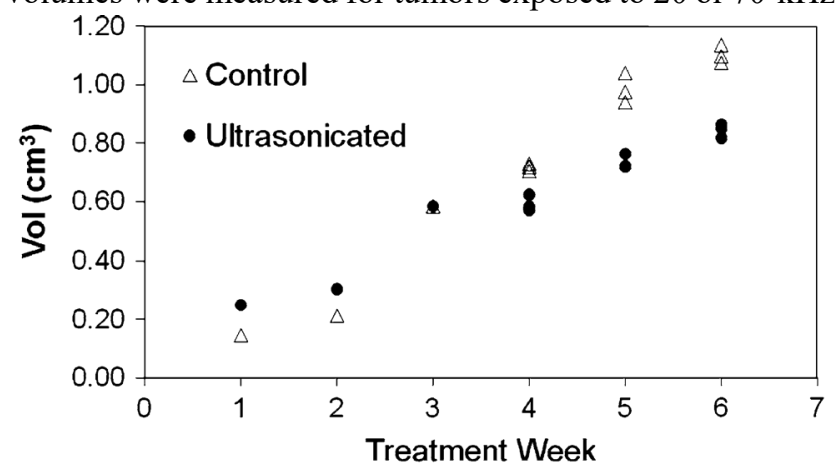

Fig. 1 Tumor volume data collected from one of the rats (from the 476$\mathrm{kHz}$ group) in the study. This graph is representative of the overall results of the study and has growth rate similar to the average of the 476-kHz group: a small, but significant decrease in the growth rate of tumors exposed in vivo to low-frequency ultrasound, after its host was infused with micellar-encapsulated doxorubicin. Key points to notice are the different initial volumes at week one and the variations in measured volumes and growth trend throughout the study. The triangles represent the measured volumes of the tumor that did not receive ultrasound. The circles represent the measured volumes of the tumor that received ultrasound. Larger tumors were measured in triplicate to increase the reliability of the statistical results

only means that in this experimental set there is no sufficient evidence to reject the null hypothesis that the growth rates at the two frequencies are the same. It is still possible that the growth rates are influenced by the frequency, but if so, any difference could not be revealed through the noise and scatter in this data set of 23 rats.

Although it is not surprising that ultrasound combined with Dox delivery from a polymeric micelle suppressed the ultrasound for $1 \mathrm{~h}$. The comparison of the final tumor volumes showed that the insonated tumors had grown less at the 4-week endpoint than tumors that were not exposed to ultrasound. However, not enough data were available to show any statistically significant difference that could be attributed to the effect of 20 versus $70-\mathrm{kHz}$ ultrasound [40]. The current project, however, is more thorough because not only did it increase the sample size, but also compared all measured tumor sizes throughout the 6 weeks of treatment and determined exponential growth rate constants for both insonated and non-insonated tumors.

We employed $15 \mathrm{~min}$ of insonation in the present study, which is less than the 60-min insonation exposure of our previous study [35]. Preliminary studies showed that $15 \mathrm{~min}$ of insonation were as effective as $60 \mathrm{~min}$ [41]. We are planning to investigate the effect of various insonation times in future studies.

One must be careful in interpreting the result that there is no statistical difference in tumor growth rates attributed to the parameter of frequency at 20 and $476 \mathrm{kHz}$. Such a result does not prove that a difference does not exist. It

tumor growth rate, it was noteworthy that the frequency of ultrasound had no statistically significant bearing on the result. In general, ultrasound at $20 \mathrm{kHz}$ (low frequency) is used for very different applications than ultrasound at 476 $\mathrm{kHz}$ (mid frequency). Low-frequency ultrasound is absorbed poorly by tissues, is more difficult to focus (because of the much larger wavelength), and is used primarily for cell disruption and for the cleaning of small objects [42]. Mid-frequency ultrasound is absorbed much more readily by tissues and is therefore used in physical therapy applications to heat tissues [43], and in therapeutic applications to ablate and destroy tissue, such as high intensity focused ultrasound (HIFU) [44]. In potential clinical applications of this technology, the frequency of application is of great importance because low-frequency ultrasound cannot be focused easily. Previous studies of transdermal drug delivery $[45,46]$ and antibiotic delivery to biofilms [47, 48] suggested that low-frequency ultrasound is much more favorable at a given power density because bubble cavitation was more pronounced at lower frequencies. The present results allow extension of this technology to mid-frequency ultrasound where focusing technology is available.

These experiments were specifically designed to investigate the effect of frequency by holding other important ultrasonic parameters constant. As described previously, the temporal average intensity was held constant at $1.0 \mathrm{~W} / \mathrm{cm}^{2}$ in both experimental sets, and the MI was held 
constant at 1.22. The MI is a measure of the likelihood and intensity of collapse cavitation. A higher value of MI is achieved at higher intensities and/or lower frequencies. Thus to match the MI values, a lower intensity was employed at $20 \mathrm{kHz}$ without any pulsing, and a much higher intensity was applied in short pulses at $476 \mathrm{kHz}$. In both cases, the MI was 1.22 , which is above the threshold for biological damage [27]. The observation that frequency makes no difference in tumor growth suppression supports the idea that the MI (which was held constant) may be a key factor in determining how much drug is delivered to the cells. Using a similar argument, it is possible that time-average intensity could also be a determining factor in tumor growth rate since that parameter was also held constant at $1.0 \mathrm{~W} / \mathrm{cm}^{2}$ in these experiments.

The observation that ultrasonic frequency (over the range studied) produced no measurable difference on tumor growth rate leads to important clinical implications. For example it is possible that future studies and clinical applications can use the most convenient ultrasonic frequency for drug delivery therapy to the tumors as long as the MI and power density are appropriate to generate positive results and yet to avoid collateral damage of healthy tissues. The effects of varying the MI and power density on tumor growth are still unknown but are the subjects of ongoing studies. A larger MI or power density could produce a stronger therapeutic effect, but that postulate remains to be tested.

This study corroborated previous studies by ourselves and others $[3,35,39]$ that there is a beneficial and synergistic effect of combining ultrasound with chemotherapy. There may be many possible reasons for this favorable synergy. Although blood with the same concentration of drug perfused both bilateral tumors, the ultrasound may have released more drug from the micelles, depositing more Dox in the insonated tumor tissue. Although such a hypothesis is consistent with many in vitro studies [5, 31-34], release from micelles has never been demonstrated in vivo. As part of a concurrent pharmacokinetics study, the tumors from this study were removed and homogenized, and the doxorubicin was extracted and quantified [49, 50]. These other studies revealed an increased concentration of drug in insonated tumors within the first $30 \mathrm{~min}$ after treatment $(\mathrm{P}=0.055)$. Thus we hypothesize that ultrasound creates a condition leading to a greater concentration of drug in the tumor [51].

Often the capillaries of tumors have enhanced permeability toward sub-micron-sized particles, similar in size to that of our micellar drug carrier $[52,53]$. Thus it is possible that drug carriers might be accumulating in the tumor due to extravasation through leaky capillaries, often called the enhanced permeation and retention (EPR) effect [54]. Ultrasound may further enhance that permeability [55, 56]. If extravasation does occur, then it would be advantageous to allow the drug-laden micelles to accumulate in the tumor for several minutes to hours before application of the ultrasound to the tumor. Future studies should vary the time after carrier injection when the ultrasound is applied in an effort to leverage the EPR effect to further enhance tumor treatment.

A third possible biological mechanism at play in our results is that ultrasound may be enhancing the permeability of the cell membrane toward drug uptake, or even perhaps toward carrier uptake. Schlicher et al. [57] demonstrated that ultrasound facilitates uptake and retention of molecules present during insonation or introduced shortly after insonation ends. Also, they showed that cells exposed to ultrasound display membrane wounds or pores that were eventually repaired $[26,57,58]$. We believe that all three biological mechanisms proposed above contribute to US enhanced tumor growth suppression.

It is also possible that, along with the lethal effect of Dox, the ultrasound lysed the cells or killed them indirectly via necrosis or stress-induced apoptosis. Low-frequency ultrasound alone at high enough intensities has been demonstrated to trigger cell-mediated death [59], but those intensities reported for cell death are much higher than those used in the experiments herein. Therefore we are not supportive for this mechanism until more supporting evidence is revealed.

While the statistical results showed that ultrasound improves the effectiveness of Dox delivery using micelles, the combined treatment failed to completely treat and cause regression of the cancerous tumors. Only 2 of the 23 tumors that received ultrasound completely regressed, and at least 1 of them is thought to have regressed mainly because of Dox therapy and the rat's immune system because the contralateral control tumor in this rat did not grow as fast as the control tumors in other rats. The other 21 insonated tumors continued growing in size, albeit at a slower rate than their contralateral control, on average.

Though this study showed that frequency has no effect on the treatment, other factors such as exposure time, MI, number of treatments per week, and drug concentration in the micelles could be manipulated to improve the effectiveness. As mentioned, mechanical index is thought to be especially important in permeating cell membranes and releasing drugs from the micelles. 


\section{Conclusions}

The combination of doxorubicin in micelles followed by the exposure to low-frequency ultrasound (at an intensity large enough to generate a high mechanical index) was effective in decreasing the tumor growth rate compared with noninsonated tumors. The tumor volumes were satisfactorily fitted to an exponential growth model where the growth rate constant for insonated tumors was $0.0402 /$ day, while the rate constant for non-insonated tumors was $0.0465 /$ day. However, different ultrasound frequencies (at the same mechanical index and time-averaged power density) showed no effect on tumor growth rate. These results implicate a key role for ultrasonic cavitation events, most probably through the release of drug from the micellar carrier, and through increased permeability of the cells and capillary walls.

Acknowledgment Funding for this research was provided by the NIH (CA98138).

Conflict of interest statement None.

\section{References}

1. Husseini GA, Pitt WG (2008) Micelles and nanoparticles inultrasonic drug and gene delivery. Adv Drug Deliv Rev 60:1137- 1152

2. Husseini GA, Pitt WG (2009) Ultrasonic-activated micellar drugdelivery for cancer treatment. J Pharm Sci (in press). doi: $10.1002 /$ jps. 21444

3. Rapoport N, Pitt WG, Sun H, Nelson JL (2003) Drug delivery inpolymeric micelles: from in vitro to in vivo. $\mathrm{J}$ Control Rel 91:85-95

4. Husseini GA, de la Diaz Rosa MA, Gabuji T, Zeng Y, Christensen DA, Pitt WG (2007) Release of doxorubicin from unstabilized and stabilized micelles under the action of ultrasound. J Nanosci Nanotech 7:1-6

5. Pruitt JD, Pitt WG (2002) Sequestration and ultrasoundinducedrelease of doxorubicin from stabilized pluronic P105 micelles. Drug Deliv 9:253-258

6. Rapoport N, Gao ZG, Kennedy A (2007) Multifunctional nanoparticles for combining ultrasonic tumor imaging and targeted chemotherapy. J Natl Cancer Inst 99:1095-1106

7. Husseini GA, Pitt WG (2008) The use of ultrasound and micellesin cancer treatment. J Nanosci Nanotechnol 8:2205-2215

8. Huang S (2008) Liposomes in ultrasonic drug and gene delivery.Adv Drug Deliv Rev 60:1167-1176

9. Frenkel V, Etherington A, Greene M, Quijano J, Xie JW, HunterF, Dromi S, Li KCP (2006) Delivery of liposomal doxorubicin (Doxil) in a breast cancer tumor model: Investigation of potential enhancement by pulsed-high intensity focused ultrasound exposure. Acad Radiol 13:469-479

10. Dromi S, Frenkel V, Luk A, Traughber B, Angstadt M, Bur M,Poff J, Xie JW, Libutti SK, Li KCP, Wood BJ (2007) Pulsed-high intensity focused ultrasound and low temperature sensitive liposomes for enhanced targeted drug delivery and antitumor effect. Clin Cancer Res 13:2722-2727

11. Kheirolomoom A, Dayton PA, Lum AFH, Little E, Paoli EE,Zheng H, Ferrara KW (2007) Acoustically-active microbubbles conjugated to liposomes: characterization of a proposed drug delivery vehicle. J Controll Release 118:275-284

12. Larina IV, Evers BM, Bartels C, Ashitkov TV, Larin KV,Esenaliev RO (2002) Ultrasound-enhanced drug delivery for efficient cancer therapy. Presented at Joint Meeting of the IEEE Engineering in Medicine and Biology Society and The Biomedical Engineering Society, Houston, TX

13. Ivanoa Y, Evers BM, Thomas R, Ashitkov TV, Esenaliev $\mathrm{RO}(2002)$ Nanoparticles and ultrasound for delivery of model macromolecular anti-cancer drugs in tumors. Presented at Joint Meeting of the IEEE Engineering in Medicine and Biology

Society and The Biomedical Engineering Society, Houston, TX

14. Crowder KC, Hughes MS, Marsh JN, Barbieri AM, Fuhrhop RW,Lanza GM, Wickline SA (2005) Sonic activation of molecularlytargeted nanoparticles accelerates transmembrane lipid delivery to cancer cells through contact-mediated mechanisms: Implications for enhanced local drug delivery. Ultrasound Med Biol 31:1693-1700

15. Larina IV, Evers BM, Ashitkov TV, Bartels C, Larin KV,Esenaliev RO (2005) Enhancement of drug delivery in tumors by using interaction of nanoparticles with ultrasound radiation. Technol Cancer Res Treat 4:217-226

16. Chumakova OV, Liopo AV, Andreev VG, Cicenaite I, Evers BM,Chakrabarty S, Pappas TC, Esenaliev RO (2008) Composition of PLGA and PEI/DNA nanoparticles improves ultrasound-mediated gene delivery in solid tumors in vivo. Cancer Lett 261:215-225

17. Hosseinkhani H, Aoyama T, Ogawa O, Tabata Y (2002) Livertargeting of plasmid DNA by pullulan conjugation based on metal coordination. J Controll Release 83:287-302

18. Hosseinkhani H, Aoyama T, Ogawa O, Tabata Y (2002) Ultrasound enhancement of in vitro transfection of plasmid DNA by a cationized gelatin. J Drug Target 10:193-204

19. Hosseinkhani H, Tabata Y (2005) Ultrasound enhances in vivotumor expression of plasmid DNA by PEG-introduced cationized dextran. J Controll Release 108:540-556

20. Aoyama T, Hosseinkhani H, Yamamoto S, Ogawa O, Tabata $Y(2002)$ Enhanced expression of plasmid DNA-cationized gelatin complex by ultrasound in murine muscle. $\mathrm{J}$ Controll Release $80: 345-356$

21. Hosseinkhani H, Kushibiki T, Matsumoto K, Nakamura T,Tabata Y (2006) Enhanced suppression of tumor growth using a combination of NK4 plasmid DNA-PEG engrafted cationized dextran complex and ultrasound irradiation. Cancer Gene Ther 13:479-489

22. Frenkel V (2008) Ultrasound mediated delivery of drugs andgenes to solid tumors. Adv Drug Deliv Rev 60:1193-1208

23. Wu JR, Nyborg WL (2008) Ultrasound, cavitation bubbles andtheir interaction with cells. Adv Drug Deliv Rev 60:1103-1116

24. Sboros V (2008) Response of contrast agents to ultrasound. AdvDrug Deliv Rev 60:1117-1136

25. Brennen CE (1995) Cavitation and bubble dynamics. OxfordUniversity Press, New York

26. Prentice P, Cuschierp A, Dholakia K, Prausnitz M, Campbell $\mathrm{P}(2005)$ Membrane disruption by optically controlled microbubble cavitation. Nat Phys 1:107-110

27. Barnett S (1998) Thresholds for nonthermal bioeffects: theoretical and experimental basis for a threshold Index. Ultrasound Med Biol 24:S41-S49 
28. Bouakaz A, Versluis M, de Jong N (2005) High-speed opticalobservations of contrast agent destruction. Ultrasound Med Biol 31:391-399

29. Husseini GA, Diaz MA, Richardson ES, Christensen DA, PittWG (2005) The role of cavitation in acoustically activated drug delivery. J Controll Release 107:253-261

30. Husseini GA, Myrup GD, Pitt WG, Christensen DA, RapoportNY (2000) Factors affecting acoustically-triggered release of drugs from polymeric micelles. J Controll Release 69:43-52

31. Husseini GA, Rapoport NY, Christensen DA, Pruitt JD, Pitt WG(2002) Kinetics of ultrasonic release of doxorubicin from Pluronic P105 micelles. Coll Surf B Biointerf 24:253-264

32. Husseini GA, Runyan CM, Pitt WG (2002) Investigating themechanism of acoustically activated uptake of drugs from Pluronic micelles. BMC Cancer 2:20

33. Marin A, Muniruzzaman M, Rapoport N (2001) Acoustic activation of drug delivery from polymeric micelles: effect of pulsed ultrasound. J Controll Release 71:239-249

34. Munshi N, Rapoport N, Pitt WG (1997) Ultrasonic activated drugdelivery from Pluronic P-105 micelles. Cancer Lett 117:1-7

35. Nelson JL, Roeder BL, Carmen JC, Roloff F, Pitt WG (2002) Ultrasonically activated chemotherapeutic drug delivery in a rat model. Cancer Res 62:7280-7283

36. Pruitt JD, Husseini G, Rapoport N, Pitt WG (2000) Stabilizationof Pluronic P-105 micelles with an interpenetrating network of N,Ndiethylacrylamide. Macromolecules 33:9306-9309

37. Jacquet P, Stuart OA, Dalton R, Chang D, Sugarbaker PH (1996) Effect of intraperitoneal chemotherapy and fibrinolytic therapy on tumor implantation in wound sites. J Surg Oncol 62:128-134

38. Martin F, Caignard A, Jeannin JF, Leclerc A, Martin M (1983) Selection by trypsin of two sublines of rat colon cancer cells forming progressive or regressive tumors. Int J Cancer 32:623627

39. Myhr G, Moan J (2006) Synergistic and tumour selective effectsof chemotherapy and ultrasound treatment. Cancer Lett 232:206213

40. Nelson JL (2002) Ultrasonically enhanced drug delivery ofdoxorubicin in vivo from stabilized pluronic micelle carriers, in chemical engineering. Brigham Young University, Provo, p 79

41. Staples BJ, Pitt WG, Schaalje B, Roeder BL (2007) Ultrasonically-assisted drug delivery in rats reduces tumor growth. Presented at Controlled Release Society, Long Beach, CA

42. Pitt WG, Husseini GA, Staples BJ (2004) Ultrasonic drugdelivery - a general review. Expert Opin Drug Deliv 1:37-56

43. Draper DO, Castel JC, Castel D (1995) Rate of temperatureincrease in human muscle during $1 \mathrm{MHz}$ and $3 \mathrm{MHz}$ continuous ultrasound. J Orthop Sports Phys Ther 22:142-150

44. Kennedy JE, Ter Haar GR, Cranston D (2003) High intensityfocused ultrasound: surgery of the future? Br J Radiol 76:590- 599

45. Mitragotri S, Blankschtein D, Langer R (1995) Ultrasoundmediated transdermal protein delivery. Science 269:850-853

46. Mitragotri S, Kost J (2004) Low-frequency sonophoresis. Areview. Adv Drug Deliv Rev 56:589-601

47. Qian Z, Sagers RD, Pitt WG (1997) The effect of ultrasonicfrequency upon enhanced killing of P. aeruginosa biofilms. Ann Biomed Eng 25:69-76

48. Peterson RV, Pitt WG (2000) The effect of frequency and powerdensity on the ultrasonically-enhanced killing of biofilmsequestered Escherichia coli. Coll Surf B Biointerf $17: 219-227$
49. Staples BJ (2007) Pharmacokinetics of ultrasonicallyreleased,micelle-encapsulated doxorubicin in the rat model and its effect on tumor growth. Chemical Engineering Department, Brigham Young University, Provo, UT

50. Staples BJ, Roeder BL, Pitt WG (2006) Doxorubicin delivery torat tissues using polymeric micelles and ultrasound. Presented at Annual Meeting of the Society for Biomaterials. Pittsburgh, PA, USA

51. Staples BJ, Roeder BL, Husseini GA, Badamjav O, Schaalje GB,Pitt WG (2009) Distribution of doxorubicin in rats subjected to ultrasonic cancer therapy (in review)

52. Tanaka T, Shiramoto S, Miyashita M, Fujishima Y, Kaneo $\mathrm{Y}(2004)$ Tumor targeting based on the effect of enhanced permeability and retention (EPR) and the mechanism of receptormediated endocytosis (RME). Int J Pharm 277:39-61

53. Seymour LW (1992) Passive tumor targeting of soluble macromolecules and drug conjugates. Crit Rev Ther Drug Carrier Syst 9:135-187

54. Reddy LH (2005) Drug delivery to tumours: recent strategies. JPharm Pharmacol 57:1231-1242

55. Kruskal J, Goldberg S, Kane R (2001) Novel in vivo use ofconventional ultrasound to guide and enhance molecular delivery and uptake into solid tumors. Presented at Annual Meeting of the Radiological Society of North America, Chicago, IL

56. Kruskal J, Miner B, Goldberg SN, Kane RA (2002) Optimizationof conventional clinical ultrasound parameters for enhancing drug delivery into tumors. Radiology 225:587-588

57. Schlicher RK, Radhakrishna H, Tolentino TP, Apkarian RP,Zarnitsyn V, Prausnitz MR (2006) Mechanism of intracellular delivery by acoustic cavitation. Ultrasound Med Biol 32:915-924

58. Hallow DM, Mahajan AD, Prausnitz MR (2007) Ultrasonicallytargeted delivery into endothelial and smooth muscle cells in ex vivo arteries. J Controll Release 118:285-293

59. Nyborg WL (2001) Biological effects of ultrasound: developmentof safety guidelines. Part II: General review. Ultrasound Med Biol 27:301-333 